\title{
FRACTURES OF THE TIBIAL SPINE IN CHILDREN
}

\author{
AN EVALUATION OF KNEE STABILITY
}

MAUREEN P. BAXTER, JAMES J. WILEY

From the University of Ottawa

\begin{abstract}
Forty-five patients with fractures of the tibial spine were reviewed 3 to 10 years after injury in order to determine the degree of residual laxity of the cruciate or collateral ligaments.

After fractures which had been partially or completely displaced, some anterior cruciate laxity was evident, even if patients were asymptomatic. It was also found that an anatomical reduction did not prevent either laxity or some loss of full extension of the knee.
\end{abstract}

The tibial spine or intercondylar eminence is the nonarticular bony prominence between the articular surfaces of the medial and lateral plateaux and serves as an attachment for the cruciate ligaments. Meyers and McKeever $(1959,1970)$ described three varieties of tibial spine fracture - undisplaced, partially displaced hingetype, and completely displaced. They did not discuss any associated ligamentous injuries, since they felt that tibial spine fractures in children were usually isolated injuries with no concomitant damage to soft tissues. There has, however, been an increasing number of reports of knee ligament injuries in children and several authors report an association with fractures of the tibial spine (Brunelli 1978; Bradley, Shives and Samuelson 1979; Clanton et al. 1979; Hyndman and Brown 1979).

The most significant aspect of a fracture of the tibial spine is that, theoretically, it might disturb the integrity of the cruciate ligaments. Some authors report that the drawer tests reveal no residual laxity at late follow-up (Bradley et al. 1979; Molander, Wallin and Wiksted 1981) but others report a variable incidence of laxity (Clanton et al. 1979; Rinaldi and Mazzarella 1980; Zaricznyj 1977). The purpose of our study was to review retrospectively a group of patients who had sustained fractures of the tibial spine in order to see if there was any residual laxity of the cruciate or collateral ligament complexes, and to determine whether the mode of injury or method of treatment influenced the outcome.

\section{MATERIALS AND METHODS}

Eighty-two patients with fractures of the tibial spine were seen at the Children's Hospital of Eastern Ontario

M. P. Baxter, MD, FRCS(C), Assistant Professor

J. J. Wiley, MD, FRCS(C), Associate Professor

Department of Surgery, Division of Orthopaedics, University of Ottawa, Ottawa, Canada K1H $8 \mathrm{Ll}$.

Requests for reprints should be sent to Dr M. P. Baxter.

(C) 1988 British Editorial Society of Bone and Joint Surgery $0301-620 X / 88 / 2048 \$ 2.00$

J Bone Joint Surg [Br] 1988;70-B :228-30 between 1976 and $1983 ; 46$ were traceable for follow-up examination from 3 to 10 years after injury. There were nine females and 37 males. The charts and radiographs of these patients were reviewed to obtain data with regard to the mechanism of injury, the age at which it occurred, and its management. The notes written at the time of admission and at operation of ten failed to record whether there was any associated ligament injury, either because testing was painful, or because no second diagnosis was sought; the immediate follow-up notes also were inadequate in this respect. One patient was eliminated from the study because he had suffered a significant knee-ligament disruption three years after the fracture; this left 45 patients for review.

We recorded all the present knee complaints, any trauma sustained since the original injury, and the findings of clinical examination of both knees. Standard radiographs were taken (including tunnel views), and a nine-test Genucom examination (Faro Medical Technologies Inc.) of both knees was conducted, using the HighSpeed Test Protocol; the Genucom Knee Analysis System (Fig. 1) permits the examiner to perform each test while viewing an objective reading of the force applied as well as the displacement occurring simultaneously as a result of that force. The examination protocol included a procedure which compensated for the amount of soft-tissue compressibility, so that true tibial and femoral displacements could be measured separately. An accuracy of $\pm 1 \mathrm{~mm}$ for translation, $\pm 1^{\circ}$ for rotation and $\pm 1 \mathrm{lb}$ for applied force has already been documented in the performance characteristics of this device (Oliver and Raab 1984). The nine tests included in the Genucom examination were an anterior-posterior drawer test at $90^{\circ}$ and $20^{\circ}$, a dual anterior-posterior drawer test at $90^{\circ}$ degrees and $20^{\circ}$, a varus-valgus test at $20^{\circ}$, a genu recurvatum-screw home test, a medial-lateral rotation test at $80^{\circ}$, and medial and lateral pivot-shift tests. No alterations in the standard test protocol were required. All patients were examined and tested by the same examiner. 


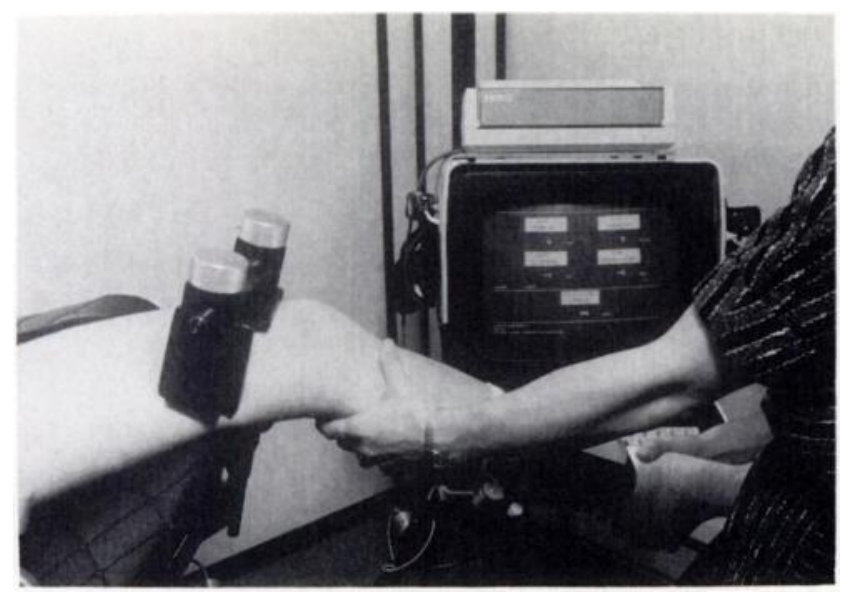

Fig. 1

The Genucom Knee Analysis System allows the examiner to perform each test while observing a simultaneous numerical display of both force and displacement.

\section{RESULTS}

The initial injury was classified from the original radiographs into the three types described by Meyers and McKeever (1959). There were eight Type 1 undisplaced fractures $(18 \%), 13$ Type 2 partially displaced $(29 \%)$, and 24 Type 3 completely displaced fractures $(53 \%) ; 42$ of the 45 injuries involved the anterior tibial spine. All three injuries involving the posterior tibial spine were completely displaced.

The displacements on the drawer tests were measured at $21 \mathrm{lb}$ of load. The varus-valgus test was measured at a torque of nine foot-pounds and the rotatory stress test at a torque of six foot-pounds. The genu recurvatum test was measured with an anterior load of $21 \mathrm{lb}$ at the foot to elicit maximum knee extension. The pivot-shift tests were measured at a load of $21 \mathrm{lb}$ and at $15^{\circ}$ of rotation.

The eight Type 1 injuries resulted in minimal residual laxity, the mean difference between the injured and uninjured knees on any test measuring no more than $1 \mathrm{~mm}(\mathrm{~s} . \mathrm{d} .=1)$. Injuries of Type 2 and 3 showed a mean difference between the knees of $3 \mathrm{~mm}$ in anterior translation at $90^{\circ}$ of flexion and of $4 \mathrm{~mm}$ at $20^{\circ}$ of flexion $($ s.d. $=1)$. The dual anterior-posterior drawer tests revealed instability in both medial and lateral compartments.

The three posterior tibial spine injuries showed a $3 \mathrm{~mm}$ difference of posterior translation between the injured and uninjured sides $(s . d .=1)$; this instability was present in both compartments. There was no clinically significant difference between the injured and uninjured knees in any other test.

Thirteen injuries (eight Type 1 and five Type 2) had been treated solely by immobilisation in plaster. No clinically significant difference in laxity between the injured and uninjured knees could be measured. Fifteen injuries (eight Type 2, seven Type 3) had been treated by closed reduction, and the remaining 17 (16 Type 3, one Type 2) had an open reduction. Both these groups showed a mean difference of $3.5 \mathrm{~mm}$ of anterior translation $(s . d .=1)$. The three posterior spine injuries showed a similar amount of posterior translation. Again, no other laxity was observed on any other test.

Seventeen of the patients had been injured while riding motorcycles, 14 on bicycles and five involved pedestrians (Fig. 2). The five pedestrians had more laxity than the other patients, with a mean of $5 \mathrm{~mm}$ of anterior translation (s.d. $=1)$; this was the only group that showed any significant valgus laxity, with a mean difference of $4 \mathrm{~mm}$ between the injured and uninjured knees $(\mathrm{s} . \mathrm{d} .=1)$.

Type 3 injuries were much more common in children aged 11 years and more (Fig. 3). However, the mean laxity resulting from these injuries was no different from that seen in younger patients.

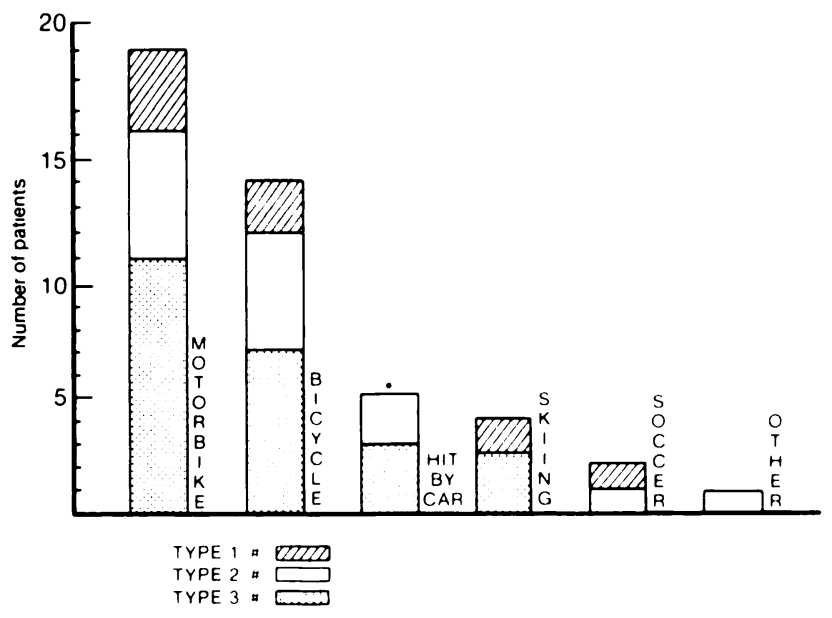

Fig. 2

Mode of injury and frequency of each type of fracture.

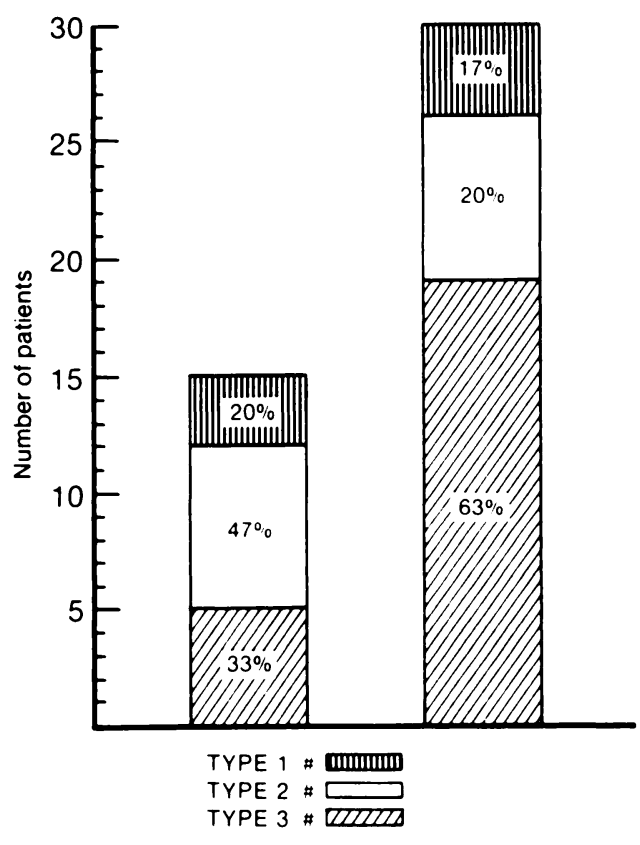

Fig. 3

Age at injury and type of fracture: the shorter column represents the children aged 10 years or less, the larger column those aged 11 or more. 
All patients had a measurable loss of extension of the injured knee (range $4^{\circ}$ to $\left.15^{\circ}\right) ; 29\left(64.4^{\circ} \%\right)$ were aware of the difference between their knees. Twenty-seven patients had a loss of more than $10^{\circ}$. Only two patients were aware of a loss of less than $10^{\circ}$.

Of the 27 patients who had an extension loss of more than $10^{\circ} 17(63 \%)$ had Type 3 injuries; nine had been treated by open reduction, seven by closed reduction, and one solely by immobilisation. Only eight Type 2 and two Type 1 injuries showed an extension loss of greater than $10^{\circ}$, while seven Type 3 injuries showed an extension loss of less than $10^{\circ}$ (Fig. 4).

\section{DISCUSSION}

This study confirms that fractures of the tibial spine may lead to permanent disturbance of cruciate ligament stability; however, except for injuries in which a pedestrian is hit by a car, no permanent disturbance of any other ligamentous complex occurs. If the medial collateral ligament is as frequently injured as some authors claim, it seems to heal with immobilisation in plaster leaving no measurable deficit.

An open reduction did not eliminate persistent loss of extension. Follow-up radiographs showed very minor changes in shape of the fractured tibial spine and no consistent change in size whatever the type of fracture or the method of treatment. This extension loss might be related to cartilaginous changes which are not evident on plain radiographs, and arthrograms could be used to delineate the shape of the intra-articular eminence accurately.

No patient in this study complained of any subjective feeling of knee instability. Although clinically 23 patients $(51 \%)$ had a positive anterior drawer test, none had a positive pivot-shift test. A change in the size and shape of the tibial spine is a reported complication of this fracture, and is thought to be due to incomplete reduction. However, undisplaced fractures also may show similar radiological changes. It has been postulated that enlargement of the tibial spine after injury is due to an increase in local blood supply during the healing stage (Ogden 1982); this structural alteration might prevent the pivot-shift test becoming positive even with a slightly lax anterior cruciate.

Seven patients had retropatellar pain in the affected knee; six, however, had similar complaints in the uninjured knee, and six were female. Only five patients reported aching in the injured knee after vigorous exercise or in humid weather; all five had undergone an open reduction. None of the remaining patients reported any knee complaints.

In conclusion, fractures of the intercondylar eminence of the tibia may result in a measurable degree of cruciate ligament laxity, although the patients are

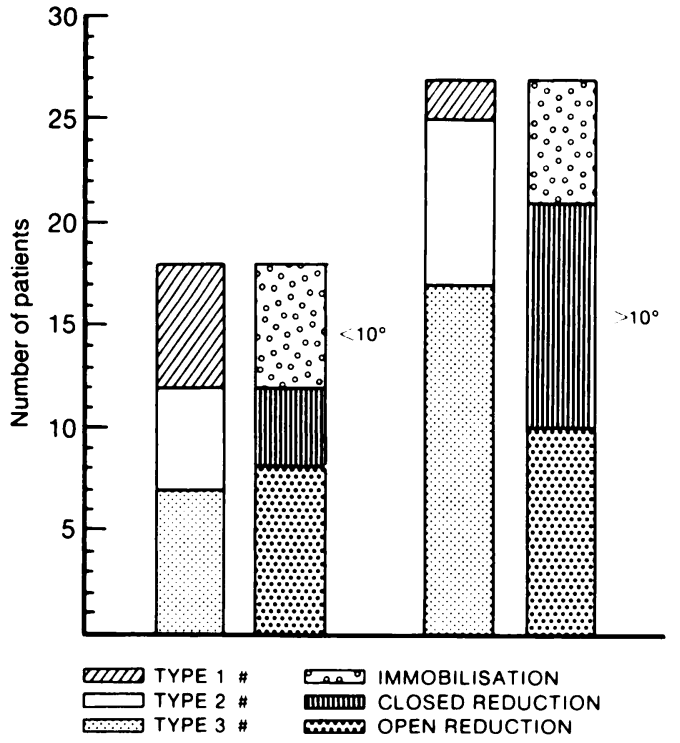

Fig. 4

Type of injury, method of treatment and loss of extension All patients had a measurable loss (range $4^{\circ}$ to $15^{\circ}$ ), and $64 \%$ were aware of this; an open reduction did not eliminate this extension loss.

generally asymptomatic. An anatomical reduction of the fracture does not eliminate the cruciate laxity or the loss of full knee extension. The overall prognosis is remarkably good even if the fracture fragment has been completely displaced. The incidence of injury to the collateral ligaments could not be evaluated by this study, and an accurate objective method of assessing these injured knees in the acute stage might be valuable.

\section{REFERENCES}

Bradley GW, Shives TC, Samuelson KM. Ligament injuries in the knees of children. J Bone Joint Surg [Am] 1979;61-A:588-91.

Brunelli G. Fractures of the intercondylar tibial eminence. Ital J Orthop Traumatol 1978;4:5-12.

Clanton TO, DeLee JC, Sanders B, Neidre A. Knee ligament injuries in children. J Bone Joint Surg [Am] 1979;61-A:1195-201.

Hyndman JC, Brown DC. Major ligamentous injuries of the knee in children. J Bone Joint Surg [Br] 1979;61-B:245.

Meyers MH, McKeever FM. Fracture of the intercondylar eminence of the tibia. J Bone Joint Surg [Am] 1959;41-A:209-22.

Meyers MH, McKeever FM. Fracture of the intercondylar eminence of the tibia. J Bone Joint Surg [Am] 1970;52-A:1677-84.

Molander M-L, Wallin G, Wikstad I. Fracture of the intercondylar eminence of the tibia: a review of 35 patients. $J$ Bone Joint Surg [Br] 1981 ;63-B:89-91.

Ogden JA. Skeletal injury in the child. Philadelphia: Lea \& Febiger, 1982.

Oliver JH, Raab S. A new device for in vivo knee stability measurement: the Genucom knee analysis system. Montreal FAR Orthopaedics Inc., 1984; Newsletter Vol. 1, No. 2.

Rinaldi E, Mazzarella F. Isolated fracture-avulsions of the tibial insertions of the cruciate ligaments of the knee. Ital J Orthop Traumatol 1980;6:77-83.

Zaricznyj B. Avulsion fracture of the tibial eminence: treatment by open reduction and pinning. $J$ Bone Joint Surg [Am] 1977;59-A : 1111-4. 